\title{
Performance of the GRACE 2.0 and EPICOR risk scores for predicting 1-year postdischarge mortality in Vietnamese patients with acute coronary syndrome
}

Thang Nguyen ${ }^{1 *}$,

Phuong Thi Bich $\mathrm{Vo}^{2}$, Hieu Thi Minh Huynh², An Viet Tran', Ca Ho Mai Tran ${ }^{1}$, Mai Tuyet $\mathrm{Vi}^{1}$, Thu Tran Anh Truong1, Thao Huong Nguyen ${ }^{2}$, Katja Taxis ${ }^{4}$

1 Department of Pharmacology and Clinical Pharmacy, Faculty of Pharmacy, Can Tho University of Medicine and Pharmacy, Can Tho, Vietnam

2 Department of Clinical Pharmacy, School of Pharmacy, University of Medicine and Pharmacy at Ho Chi Minh City, Ho Chi Minh City, Vietnam

3 Department of Internal Medicine, Faculty of Medicine, Can Tho University of Medicine and Pharmacy, Can Tho, Vietnam

${ }^{4}$ Unit of Pharmacology Therapy, Epidemiology \& Economics, Groningen Research Institute of Pharmacy, University of Groningen, Groningen, The Netherlands

*Corresponding author:

Thang Nguyen

nthang@ctump.edu.vn

Keywords:

GRACE 2.0, EPICOR, mortality risk scores, acute coronary syndrome, Vietnamese.

\begin{abstract}
We aimed to determine the performance of the Global Registry of Acute Coronary Events (GRACE) risk score version 2.0, and the long-term follow up of antithrombotic management patterns in acute coronary syndrome patients (EPICOR) risk score for predicting 1-year postdischarge mortality in patients with acute coronary syndrome (ACS) in Vietnam. A prospective cohort study was conducted on 455 patients who were discharged with an ACS diagnosis from two hospitals in Can Tho, and one in Ho Chi Minh City. Online simplified EPICOR and GRACE 2.0 calculators were used to assess the expected risk of death. We compared the performance of predicting 1-year mortality between GRACE 2.0 and EPICOR risk scores by using the Hosmer-Lemeshow test, the area under the curve (AUC), and the De-Long test. The proportions of low-risk group, moderate-risk group, and high-risk group for GRACE were $31 \%, 42 \%$, and $27 \%$, respectively. Actual mortality rates for the low, medium and high-risk groups were 3.6\%, 9.4\%, and $19.4 \%$. The proportions of low-risk and high-risk patients for EPICOR were $92.0 \%$ and $8.0 \%$, respectively. The actual mortality rate of the high-risk group was $31.4 \%$, and of the low-risk group $8.6 \%$. Hosmer-Lemeshow test results of the GRACE 2.0 and EPICOR were 0.337 and 0.001 , respectively. The AUC results of the GRACE 2.0 and EPICOR were 0.703 and 0.752 respectively, and the De-Long test with $p=0.0532$. In conclusion, the GRACE 2.0 was better than EPICOR in predicting 1-year postdischarge mortality in Vietnamese patients with ACS.
\end{abstract}

\section{INTRODUCTION}

Ischemic heart disease (IHD) is the major cause of mortality worldwide, with an increasing trend from 7.3 million in 2007 to 8.93 million in $2017^{1}$. IHD remains a health challenge for the public in developing countries. Acute coronary syndrome (ACS) is the main factor that leads to IHD deaths ${ }^{2}$. For ACS treatment international guidelines recommended using a combination of antiplatelet agents, anticoagulants, beta-blockers, angiotensin-converting enzyme inhibitors, and angiotensin-receptor blockers ${ }^{3-5}$. The pharmacological medication significantly reduced the in-hospital and postdischarge mortality of $\mathrm{ACS}^{6-8}$. However, ACS patients had
Pharmaceutical Sciences Asia (C) 2021 by Faculty of Pharmacy, Mahidol University, Thailand is licensed under CCBY-NC-ND 4.0. To view a copy of this license, visit https:// www.creativecommons.org/ licenses/by-nc-nd/4.0 
different medical histories, physical examinations, and electrocardiographic and biochemical cardiac marker data.These factors also affect ACS risk stratification.

ACS risk stratification plays an important role in disease management ${ }^{4}$. It helps physicians to classify ACS patients as well as to choose an appropriate management strategy to limit shortterm and long-term events ${ }^{5}$. There are many models of risk stratification for ACS. The GRACE score is an effective model to predict inhospital and postdischarge deaths ${ }^{9-13}$. Until now, the GRACE score has been the most popular model and is recommended in international guidelines ${ }^{4,14}$. The GRACE score has 2 versions. The GRACE 1.0 was designed in 2000 to estimate in-hospital and 6-month postdischarge mortality9, ${ }^{10}$. The GRACE 2.0 was updated by Fox KA et al. to heighten the performance of GRACE 1.0. A more modern tool, the GRACE 2.0 is more accurate than the old version in predicting short and long-term mortality ${ }^{11,12}$.

The EPICOR is a new risk score, designed to calculate one-year mortality in survivors ${ }^{15,16}$. The validated research of the EPICOR score is a new study conducted on an international cohort $^{16}$. There have previously been no studies to estimate the performance of EPICOR in Vietnam. In this study we compared the GRACE 2.0 and EPICOR risk scores on a Vietnamese cohort. According to S. Pocock et al., it would be useful to compare directly the GRACE 2.0 and EPICOR risk models in an independent cohort of ACS patients followed after hospital discharge ${ }^{16}$. Therefore, the aim of this study was to determine the performance of GRACE 2.0 and EPICOR risk scores for predicting 1-year postdischarge mortality in Vietnamese patients with ACS.

\section{MATERIALS AND METHODS}

\subsection{Study population}

This prospective cohort study was conducted in patients discharged with a diagnosis of ACS from two public hospitals in Can Tho City and one public hospital in Ho Chi Minh City. We calculated the sample size for estimating a single proportion of the high-risk group in the study population with $5 \%$ absolute precision and $95 \%$ confidence. Assuming a highrisk group proportion of $50 \%$ and a $15 \%$ loss to follow-up, we should recruit at least 443 patients. Patients included as eligible were admitted between January and October 2015 and survived during hospitalization with one of the following discharge diagnoses (according to coding of the International Classification of Diseases, $10^{\text {th }}$ revision (ICD-10)): unstable angina (I20.0), acute myocardial infarction (I21) consisting of non-ST elevation myocardial infarction (NSTEMI) and ST-elevation myocardial infarction (STEMI), or subsequent myocardial infarction (I22) ${ }^{17}$, after receiving a complete treatment. Patients were followed for one year after discharge. The follow-up period ended in October 2016. We excluded patients for whom relevant data were missing or who had initially been admitted to another hospital. The research was approved by the institutional review boards and is in line with Vietnamese regulations. For all participants the relevant data were recorded, and the mortality risk calculated and classified by GRACE and EPICOR risk scores.

\subsection{Study design and data analysis}

The GRACE 2.0 score was calculated by assigning the appropriate number of points for each of the 8 factors: age, heart rate, systolic blood pressure, serum creatinine concentration or renal failure, cardiac arrest at admission, STsegment deviation, elevated cardiac biomarkers of necrosis, and Killip class or diuretic usage ${ }^{13}$. We calculated by using an online calculator http://www.gracescore.org/WebSite/WebVersio n.aspx. Patients were classified into three risk groups: low, medium and high-risk groups. The GRACE 2.0 score was categorized using common cut-off points: $<4 \%$ (low-risk), $4 \%-12 \%$ (medium risk) and $>12 \%$ (high-risk) ${ }^{18}$.

The EPICOR score consists of the following factors: age, gender, ACS subtype, ejection fraction at hospital admission, serum creatinine at admission, hemoglobin and fasting blood glucose at admission, interventions during admission, EuroQol-5D (EQ-5D) score upon discharge (to characterize current health status), cardiac complications in hospital, history of chronic obstructive pulmonary disease (COPD) or other chronic lung disease, history of peripheral vascular disease, and on diuretic at discharge ${ }^{16}$. The EPICOR score was calculated using http:// www.acsrisk.org/calculator. Patients were divided into 6 groups according to risk: groups 1 - 4 were regarded as low-risk (mortality risk $<6.3 \%$ ), group 5 (mortality risk $>6.3 \%$ ) and group 6 (mortality risk $>18.2 \%$ ) were regarded as highrisk $^{16}$. 


\subsection{Statistical analysis}

After classifying the risk of death in 1 year by GRACE and EPICOR risk scores, we evaluated the accuracy of two risk scores by two indicators: calibration and discrimination. The calibration was described using the HosmerLemeshow test ${ }^{19}$. A $p$-value less than 0.05 was considered to be a statistically significant difference between the prediction and actual rate, indicating a poor prediction capacity of the model. The assessment of discrimination capacity was calculated by the $\mathrm{AUC}^{20}$. The AUC of a model with good discrimination was more than 0.7. We used the De-Long test to compare the AUC for the two risk scores and determine whether there was any difference between the two scores ${ }^{21}$. Statistical analysis was performed using SPSS 22, MedCalc version 18.5, and Microsoft Excel 2010.

\section{RESULTS}

A total of 455 patients with ACS were included in the analysis. Their mean age was $67.6 \pm 13$ years (range 55 to 81 years), and $79.9 \%$ had health insurance. The majority of patients were male $(58.9 \%)$ and had hypertension $(77.6 \%)$. One hundred and eighty-seven $(41.1 \%)$ patients underwent percutaneous coronary intervention (PCI) or coronary artery bypass grafting (CABG), and two hundred and sixty-eight $(58.9 \%)$ had no invasive procedure. The majority of patients had a discharge diagnosis of unstable angina and non-ST-segment elevation ACS (NSTEMI) (76.9\%) (Table 1).

Table 1. Characteristics of the study population.

\begin{tabular}{lcc}
\hline & $\begin{array}{c}\text { Number of patients } \\
(\mathbf{N}=\mathbf{4 5 5})\end{array}$ & $\begin{array}{c}\text { Percentage } \\
(\mathbf{\%})\end{array}$ \\
\hline Description & & 58.2 \\
Age $\geq 65$ & 265 & 58.9 \\
Male & 268 & 79.9 \\
Health Insurance & 362 & 10.3 \\
Number of patients died & 47 & 41.1 \\
\hline In-hospital invasion & & 58.9 \\
\hline PCI or CABG & 187 & \\
Non-invasive & 268 & 76.9 \\
\hline Discharge diagnosis & & 23.1 \\
\hline Unstable angina/NSTEMI & 350 & 77.6 \\
STEMI & 105 & 24.2 \\
\hline Medical history and comorbidities & & 19.3 \\
Hypertension & 353 & 14.3 \\
Dyslipidemia & 110 & 7.3 \\
Diabetes & 88 & 35.4 \\
Heart failure & 65 & 33 \\
Renal failure & 161 & \\
Smoking & & \\
\hline
\end{tabular}

ACS, acute coronary syndrome; CABG, coronary artery bypass grafting; NSTEMI, non-ST-segment elevation myocardial infarction; PCI, percutaneous coronary intervention; STEMI, ST-segment elevation myocardial infarction.

We compared some characteristics of the groups of those patients who died and those who survived (Table 2). In the group of patients who died, the majority were $>65$ years old $(80.9 \%)$. The rate of mortality in patients with percutaneous coronary intervention (PCI) or coronary artery bypass grafting (CABG) was lower than in patients who had not undergone invasive procedures. The rate of heart failure (34\%) and renal failure (23.4\%) was significantly higher in patients who died than in the group who survived. The proportion of hypertension $(89.4 \%)$ tended to be statistically higher in the group who died. Table 2 gives an insight into the risk factors of ACS.

The proportion of low-risk, moderaterisk, and high-risk groups for GRACE was $31 \%$, $42 \%$, and $27 \%$ respectively. The actual mortality rates for the low, medium and high-risk groups were $3.6 \%, 9.4 \%$, and $19.4 \%$. The proportion of low-risk and high-risk patients for EPICOR was $92.0 \%$ and $8.0 \%$, respectively. The actual mortality rate of the high-risk group was $31.4 \%$ and of the low-risk group $8.6 \%$. The proportion of risk categories for the GRACE 2.0 and EPICOR scores is shown in Table 3. The results of the performance of the GRACE 2.0 and the EPICOR are shown in Figure 1 and Table 4. 
Table 2. Characteristics of death and survival patients.

\begin{tabular}{|c|c|c|c|}
\hline & $\begin{array}{c}\text { Group of death patients } \\
n(\%)\end{array}$ & $\begin{array}{c}\text { Group of survival } \\
\text { patients } \\
\text { n }(\%)\end{array}$ & p-value \\
\hline Number of patients & $47(10.3)$ & $408(89.7)$ & \\
\hline \multicolumn{4}{|l|}{ Characteristic } \\
\hline Older than 65 & $38(80.9)$ & $227(55.6)$ & 0.001 \\
\hline Male & $26(55.3)$ & $242(59.3)$ & 0.598 \\
\hline Health Insurance & $38(80.9)$ & $324(79.8)$ & 0.865 \\
\hline \multicolumn{4}{|l|}{ In-hospital invasion } \\
\hline PCI or $\mathrm{CABG}$ & $9(19.1)$ & $178(43.6)$ & 0.001 \\
\hline Non-invasive & $38(80.9)$ & $230(56.4)$ & 0.001 \\
\hline \multicolumn{4}{|l|}{ Discharge diagnosis } \\
\hline Unstable angina/ NSTEMI & $40(85.1)$ & $310(76.0)$ & 0.160 \\
\hline STEMI & $7(14.9)$ & $98(24.0)$ & 0.160 \\
\hline \multicolumn{4}{|c|}{ Medical history and comorbidities } \\
\hline Hypertension & $42(89.4)$ & $311(76.2)$ & 0.041 \\
\hline Dyslipidemia & $6(12.8)$ & $104(25.5)$ & 0.054 \\
\hline Diabetes & $10(21.3)$ & $78(19.1)$ & 0.723 \\
\hline Heart failure & $16(34.0)$ & $49(12.0)$ & $<0.001$ \\
\hline Renal failure & $11(23.4)$ & $22(5.4)$ & $<0.001$ \\
\hline Smoking & $17(36.2)$ & $144(35.3)$ & 0.905 \\
\hline
\end{tabular}

ACS, acute coronary syndrome, CABG, coronary artery bypass grafting; NSTEMI, non-ST-segment elevation myocardial infarction; PCI, percutaneous coronary intervention; STEMI, ST-segment elevation myocardial infarction.

Table 3. The proportion of risk categories for GRACE 2.0 and EPICOR risk scores.

\begin{tabular}{lcc}
\hline & $\begin{array}{c}\text { GRACE 2.0 score } \\
\text { n }(\boldsymbol{\%})\end{array}$ & $\begin{array}{c}\text { EPICOR score } \\
\text { n (\%) }\end{array}$ \\
\hline Group of risk category $(\mathbf{N}=\mathbf{4 5 5})$ & & \\
\hline Low risk & $139(31.0)$ & $420(92.0)$ \\
Intermediate risk & $192(42.0)$ & - \\
High risk & $124(27.0)$ & $35(8.0)$ \\
\hline Actual mortality rate & & \\
\hline Low risk & $5(3.6)$ & $36(8.6)$ \\
Intermediate risk & $18(9.4)$ & - \\
High risk & $24(19.4)$ & $11(31.4)$ \\
\hline
\end{tabular}

GRACE, global registry of acute coronary event; EPICOR, long - term follow up of antithrombotic management patterns in acute coronary syndrome patients.

Table 4. The calibration and discrimination capacity of GRACE 2.0 and EPICOR in one-year mortality ACS prediction.

\begin{tabular}{|c|c|c|c|}
\hline & $\begin{array}{l}\text { Hosmer - Lemeshow } \\
\text { (p) test } \\
\text { (The calibration) }\end{array}$ & $\begin{array}{c}\text { The area under the curve AUC } \\
(95 \% \text { CI }) \\
\text { (The discrimination) }\end{array}$ & De - Long test \\
\hline GRACE 2.0 & 0.337 & $0.703(p<0.001)$ & $p=0.0532$ \\
\hline EPICOR & 0.001 & $0.752(p<0.001)$ & $p=0.0532$ \\
\hline
\end{tabular}

AUC, the area under the curve; 95\% CI, confidence interval; GRACE, global registry of acute coronary event; EPICO, long - term follow up of antithrombotic management patterns in acute coronary syndrome patients.

\section{DISCUSSION}

Generally, this prospective cohort study gave an insight into the performance of EPICOR as compared with GRACE 2.0. The mean patient age in this sample $(67.6 \pm 13)$ was similar to that in another study in The United States ${ }^{3}$. Age is an important risk factor in GRACE and EPICOR scores ${ }^{11,16}$; in fact, Pocock et al. demonstrated age to be the leading factor causing the mortality in the EPICOR score ${ }^{16}$. The higher age led to a higher rate of mortality in $\mathrm{ACS}^{22}$. The rate of survival patients who had undergone invasion procedures was significantly higher than the rate of mortality patients because the early invasive procedure reduced mortality, recurrent risk and complications $^{23,24}$. In this study, the rate of patients who had interventions tends to be significantly lower than in other research ${ }^{14,16}$ because of the economic situation of the patients 


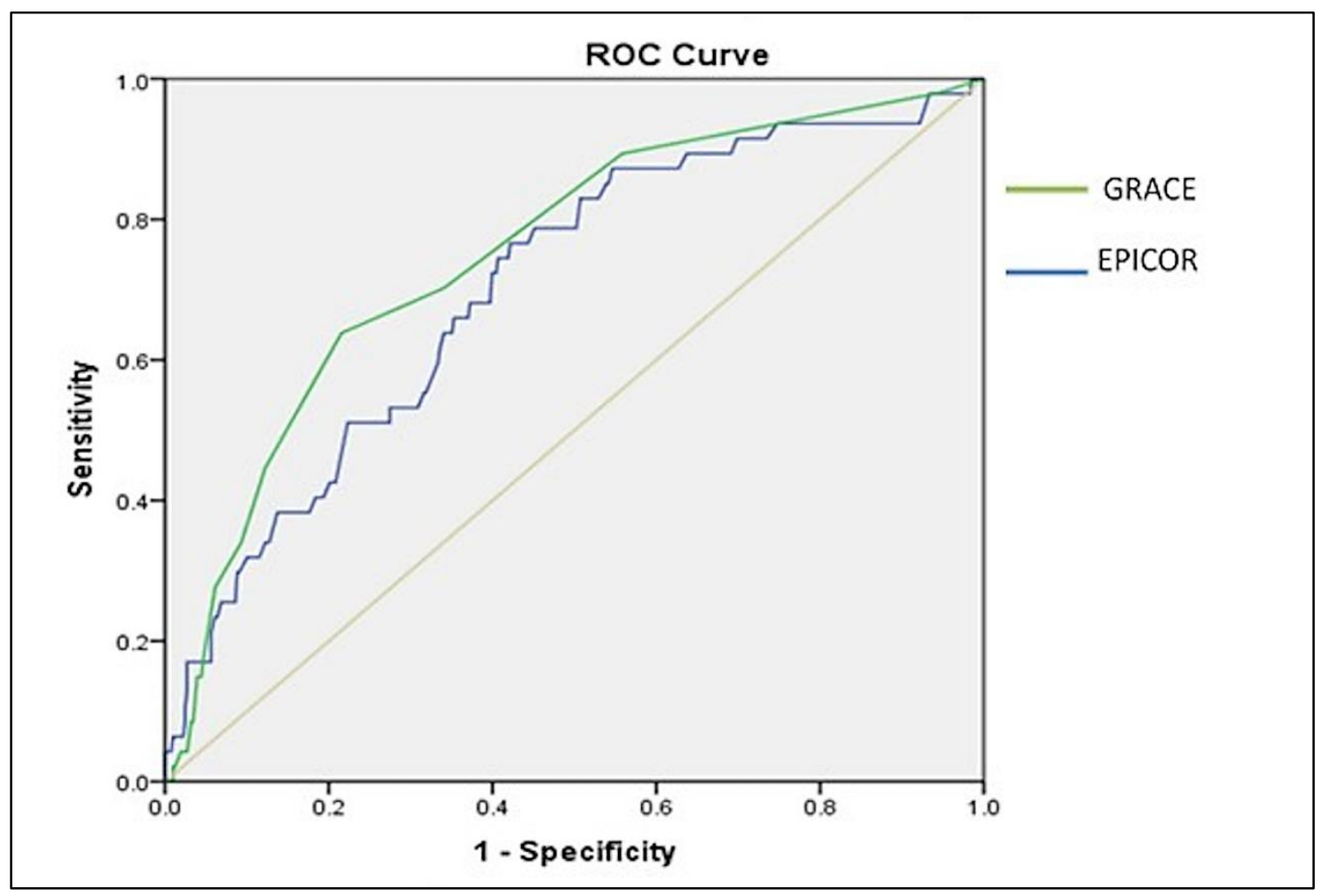

Figure 1. AUC of GRACE 2.0 and EPICOR risk cores.

and inadequate techniques for invasion in one hospital. Rates of hypertension, heart failure and renal failure in the mortality group were significantly higher than in the survivor group. Hypertension increases the risk of sudden death, coronary spasms, and coronary thrombosis ${ }^{25}$. Heart failure and renal failure are also two risk factors of ACS to calculate the GRACE 2.0 score $^{18}$. Therefore, these factors could raise the prognosis of mortality.

According to categorization of the GRACE 2.0 score, the percentages of patients in the low-risk group, the moderate-risk group, and the high-risk group were $31 \%, 42 \%$, and $27 \%$, respectively. Overall, the majority of patients was in the moderate risk groups. This result was similar to the findings of Akyuz et al. $2016^{18}$ and Bekler et al. ${ }^{26}$. According to the EPICOR score, more than $90 \%$ of patients were in the low-risk group and no more than $10 \%$ were in the highrisk group. The results of risk stratification between EPICOR and GRACE 2.0 scores were different. This was explained by the difference in the classification of the two risk scores. In addition, there were a number of different factors in the calculation of the two scores. The EPICOR score needs to investigate ejection fraction upon admission, cardiovascular complications during hospital stay, etc. ${ }^{16,18}$ Both the GRACE 2.0 and EPICOR scores showed that the actual mortality rate of patients in the high-risk group was higher than in the other groups. The basic analysis of this study showed that discrimination was not a statistical difference. However, the calibration ability of the GRACE 2.0 score was better than that of the EPICOR score.

In our study, the Hosmer-Lemeshow test results of GRACE 2.0 and EPICOR scores were 0.337 and 0.001 , respectively. The calibration compared the predicted and observed mortality rates. The results showed a satisfactory calibration capacity of the GRACE 2.0 score $(p=0.337>$ $0.05)$; thus, there was no difference between the predicted value and actual results of death events within one year after the discharge of patients with ACS. This showed the relevance of the GRACE 2.0 score for the study population. Other research has also provided evidence for the good performance of GRACE 2.0. The study of L.C. Correia et al. (2014) reported the ability to adjust the GRACE 2.0 score as $p=0.08^{22}$. In 2015, Akyuz et al. assessed the calibration ability of the score on each different risk group (classified by GRACE 2.0 score); the Hosmer-Lemeshow results of the GRACE 2.0 score for low, medium and high-risk patients were $0.66,0.75$, and 0.96 , respectively ${ }^{18}$. In contrast, our results of the EPICOR score as indicated by the HosmerLemeshow test did not correspond with the study population $(p=0.001<0.05)$. Because of the low 
calibration, the EPICOR score was not valid with this population. However, the previous study of Pocock demonstrated the excellent calibration ability of the EPICOR score ${ }^{16}$. The EPICOR score was not suitable for this study because of the sensitivity of the population used for the model sensitivity of the population with the model. Another reason was that we were not able to record the EuroQol-5D (EQ-5D) score in all patients of the sample, although this factor was allowed to be omitted ${ }^{16}$. Both GRACE 2.0 and EPICOR scores were capable of discriminating well, with AUCs of 0.703 and 0.752 , respectively. The AUC of the GRACE 2.0 score in this study was lower than that in the research of Huang et al., $2016(0.77)^{13}$. The sample sizes of the two studies were not significantly different. The difference in the prognostic capacity of the GRACE 2.0 scores between the studies may be due to the characteristics of the study population.

When comparing the discriminatory ability of the GRACE 2.0 score in this study with the original validation study of the GRACE 2.0 score $^{13}$, we found the discriminatory ability of the GRACE 2.0 score in the original study to be better $(0.83>0.703)$. This could be explained because the population in the original study was 7 times larger than our study sample. The EPICOR score had good ability to discriminate (AUC $=0.752$ ). When we compared our study with the original study - the study to establish and build the EPICOR score - the AUC of the EPICOR score in this study was lower $(0.752<0.81)^{16}$. In general, the discriminatory ability of the EPICOR score tends to be better than the GRACE 2.0 score (0.752 compared with 0.703). However, the DeLong test result had $p=0.0532$; this showed that between GRACE 2.0 and EPICOR scores there was no difference in the ability to distinguish. Based on the ability to calibrate and distinguish between GRACE 2.0 and EPICOR scores, the GRACE 2.0 was suitable for predicting mortality within one year after discharge in patients in the study cohort who had acute coronary syndrome.

Generally, GRACE 2.0 is a popular, simple, effective scale to estimate the risk of death in patients with ACS in hospital and postdischarge. The factors of GRACE 2.0 easy to collect from preclinical and clinical data of patients in hospital. Moreover, this risk score has been validated through many large-scale studies. Our study also demonstrates the good quality of this risk score about predicting one-year mortality. On the other hand, GRACE 2.0 is not specific for one-year mortality and it does not contain any information about the medical history and health status of patients at hospital discharge and postdischarge. EPICOR risk score includes these factors in the calculation, and it is specific for a one-year mortality estimation. However, these factors can be missed or incorrect because they are provided by patients. A small number of studies have validated this new risk score $^{11,12,15,16}$.

Some limitations of our study should be considered. Data were collected from only three hospitals in Vietnam. However, the sample was adequate for assessing calibration and discrimination. Results could, however, differ in different countries and with different ethnicities. Therefore, the difference in the performance of EPICOR score between this study and the research of Pocock et al. can be attributed to the study sample. The GRACE 2.0 score is just one of many risk-stratification tools. We could not conclude that this was the best model to predict one-year mortality in ACS patients. In future studies, we should compare the GRACE 2.0 score with other scores to determine the most suitable score. That we were unable to record the EuroQol-5D (EQ-5D) score in this population could explain the poor performance of the EPICOR score.

This research can be regarded as the first study to compare the GRACE 2.0 and EPICOR risk scores in the same population. Our study found the GRACE 2.0 score to be a good model for predicting one-year mortality in Vietnamese patients with ACS. In the future, to have a better assessment and find the most suitable standard for Vietnam, we need to conduct comparative research on GRACE, EPICOR and another score (such as the thrombolysis in myocardial infarction -TIMI) related to the rate of mortality in one year.

\section{CONCLUSIONS}

A significant difference was found between the performance of the GRACE 2.0 and EPICOR risk scores. In this study, the GRACE 2.0 score was more accurate for predicting one-year mortality after discharge in patients with acute coronary syndrome, compared to the EPICOR score. The GRACE 2.0 score is an effective tool for estimating one-year mortality risk in Vietnam.

\section{ACKNOWLEDGMENT}

None. 


\section{Conflict of interests}

There are no competing interests.

Funding

None to declare.

\section{Ethics approval}

None to declare.

\section{Article info:}

Received March 18, 2020

Received in revised form July 29, 2020

Accepted November 13, 2020

\section{REFERENCES}

1. GBD Causes of Death Collaborators. Global, regional, and national age-sex-specific mortality for 282 causes of death in 195 countries and territories, 1980-2017: a systematic analysis for the Global Burden of Disease Study 2017. Lancet 2018;392(10159):1736-88.

2. Amsterdam EA, Wenger NK, Brindis RG, Casey DE Jr, Ganiats TG, Holmes DR Jr, et al. 2014 AHA/ACC Guideline for the management of patients with non-STelevation acute coronary syndromes: a report of the American College of Cardiology/American Heart Association Task Force on Practice Guidelines. J Am Coll Cardiol. 2014;64(24):e139-e228.

3. Roffi M, Patrono C, Collet JP, Mueller C, Valgimigli M, Andreotti F, et al. 2015 ESC guidelines for the management of acute coronary syndromes in patients presenting without persistent ST-segment elevation: task force for the Management of Acute Coronary Syndromes in patients presenting without persistent STsegment elevation of the European Society of Cardiology (ESC). Eur Heart J 2016; 37(3):267-315.

4. Spinler SA. Acute coronary syndromes. In: Dunsworth TS, Richardson MM, Cheng JWM, Chessman KH, Hume AL, Hutchison LC, et al, eds. Pharmacotherapy Self-assessment Program. Book 1. Cardiology, $6^{\text {th }}$ edition. Kansas City: American College of Clinical Pharmacy. 2007:59-83.

5. Yan AT, Yan RT, Tan M, Huynh T, Soghrati K, Brunner LJ, et al. Optimal medical therapy at discharge in patients with acute coronary syndromes: temporal changes, characteristics, and 1-year outcome. Am Heart J. 2007; 154(6):1108-15

6. Fox KA, Steg PG, Eagle KA, Goodman SG, Anderson FA Jr, Granger CB, et al. Decline in rates of death and heart failure in acute coronary syndromes, 1999-2006. JAMA. 2007; 297(17):1892-900.

7. Jernberg T, Johanson P, Held C, Svennblad B, Lindbäck $\mathrm{J}$, Wallentin $\mathrm{L}$, et al. Association between adoption of evidence-based treatment and survival for patients with ST-elevation myocardial infarction. JAMA. 2011;305(16):1677-84.

8. Fox KA, Dabbous OH, Goldberg RJ, Pieper KS, Eagle KA, Van de Werf F, et al. Prediction of risk of death and myocardial infarction in the six months after presentation with acute coronary syndrome: prospective multinational observational study (GRACE). BMJ. 2006;333(7578):1091.

9. Eagle KA, Lim MJ, Dabbous OH, Pieper KS, Goldberg RJ, Van de Werf F, et al. A validated prediction model for all forms of acute coronary syndrome estimating the risk of 6-month postdischarge death in an international registry. JAMA. 2004; 291(22):2727-33.

10. Fox KA, Fitzgerald G, Puymirat E, Huang W, Carruthers K, Simon T, et al. Should patients with acute coronary disease be stratified for management according to their risk? Derivation, external validation and outcomes using the updated GRACE risk score. BMJ Open. 2014;4(2):e004425.

11. Granger CB, Goldberg RJ, Dabbous O, Pieper KS, Eagle $\mathrm{KA}$, Cannon CP, et al. Predictors of hospital mortality in the global registry of acute coronary events. Arch Intern Med. 2003;163(19): 2345-53.

12. Huang W, FitzGerald G, Goldberg RJ, Gore J, McManus $\mathrm{RH}$, Awad H, et al. Performance of the GRACE Risk Score 2.0 Simplified Algorithm for Predicting 1-Year Death After Hospitalization for an Acute Coronary Syndrome in a Contemporary Multiracial Cohort. Am J Cardiol. 2016;118(8):1105-10.

13. Tra J, van der Wulp I, Appelman Y, de Bruijne MC, Wagner C. Adherence to guidelines for the prescription of secondary prevention medication at hospital discharge after acute coronary syndrome: a multicentre study. Neth Heart J. 2015; 23(4):214-21.

14. Bueno H, Danchin N, Tafalla M. EPICOR (long-tErm follow-up of antithrombotic management Patterns In acute CORonary syndrome patients) study: Rationale, design, and baseline characteristics. Am Heart J. 2013; 165(1):8-14

15. Pocock S, Bueno H, Licour M, Medina J, Zhang L, Annemans L, et al. Predictors of one-year mortality at hospital discharge after acute coronary syndromes: a new risk score from the EPICOR (long-tErm follow up of antithrombotic management patterns in acute CORonary syndrome patients) study. Eur Heart J Acute Cardiovasc Care. 2015;4(6):509-17.

16. World Health Organization. International statistical classification of diseases and related health problems 10th revision (ICD-10)-WHO Version for 2016.

17. Akyuz S, Yazici S, Bozbeyoglu E, Onuk T, Yildirimturk $\mathrm{O}$, Karacimen D, et al. Validity of the updated GRACE risk predictor (version 2.0) in patients with non-STelevation acute coronary syndrome. Rev Port Cardiol. 2016;35(1), 25-31.

18. Lemeshow S, Hosmer DW Jr. A review of goodness of fit statistics for use in the development of logistic regression models. Am J Epidemiol. 1982;115(1):92-106.

19. Hanley JA, McNeil BJ. The meaning and use of the area under a receiver operating characteristic (ROC) curve. Radiology. 1982;143(1):29-36.

20. DeLong ER, DeLong DM, Clarke-Pearson DL. Comparing areas under two or more correlated receiver operating characteristics curves: a nonparamentric approach. Biometrics. 1988;44(3):837-45.

21. Correia LC, Freitas R, Bittencourt AP, Souza AC, Almeida MC, Leal J, et al. Prognostic value of GRACE scores versus TIMI score in acute coronary syndromes. Arq Bras Cardiol. 2010;94(5),613-19.

22. Hasdai D, Behar S, Wallentin L, Danchin N, Gitt AK, Boersma E, et al. A prospective survey of the characteristics, treatments and outcomes of patients with acute coronary syndromes in Europe and the Mediterranean basin; the Euro Heart Survey of Acute Coronary Syndromes (Euro Heart Survey ACS). Eur Heart J. 2002;23(15):1190-201.

23. Fragmin and Fast Revascularisation during Instability in Coronary Artery Disease Investigators. Invasive compared with non-invasive treatment in unstable 
coronary-artery disease: FRISC II prospective randomised multicentre study. Lancet. 1999;354 (9180):708-15.

24. Wallentin L, Lagerqvist B, Husted S, Kontny F, Ståhle E, Swahn E. Outcome at 1 year after an invasive compared with a non-invasive strategy in unstable coronary-artery disease: the FRISC II invasive randomised trial. FRISC II Investigators. Fast Revascularisation during Instability in Coronary artery disease. Lancet. 2000;356(9223):9-16.
25. Julius S. Corcoran Lecture. Sympathetic hyperactivity and coronary risk in hypertension. Hypertension. 1993;21(6),886-93.

26. Bekler A, Altun B, Gazi E, Temiz A, Barutçu A, Güngör Ö, et al. Comparison of the GRACE risk score and the TIMI risk index in predicting the extent and severity of coronary artery disease in patients with acute coronary syndrome. Anatol J Cardiol. 2015;15(10),801-6. 